\title{
Implantierbares Sensorsystem mit biokompatibler Verkapselung
}

\author{
Carola Jorsch ${ }^{1}$, David Ulkoski ${ }^{2}$, Carmen Scholz ${ }^{2}$, Margarita Guenther ${ }^{1}$ und Gerald Gerlach ${ }^{1}$ \\ ${ }^{1}$ Institut für Festkörperelektronik, Technische Universität Dresden, Dresden, Deutschland \\ ${ }^{2}$ Department of Chemistry, University of Alabama in Huntsville, Huntsville AL, USA \\ Kontakt: Carola.Jorsch@tu-dresden.de
}

\section{Zusammenfassung}

Für die kontinuierliche Erfassung von physiologischen Parametern im Rahmen der Therapie von Stoffwechselkrankheiten, wie z.B. Diabetes mellitus, sind implantierbare drahtlose SensorarrayMikrosysteme eine Herausforderung. Die sensiblen Messsysteme bestehen aus mehreren Hydrogelbasierten piezoresistiven Sensoren, die eine Inline-Überwachung physiologischer Parameter in menschlicher Körperflüssigkeit ermöglichen. Zusätzlich zur Untersuchung und Modellierung der Quellkinetik und der Diffusionsprozesse in Hydrogelen wird die Biokompatibilität des entwickelten Mikrosystems in den Vordergrund gestellt. Im Hinblick auf die vorgesehene Verwendung wurde deshalb für die Einhausung des Sensors die Medizinprodukte-Norm DIN EN ISO 10993-5:2009 herangezogen. Die hier verwendete mehrschichtige Verkapselung des implantierbaren Sensorarray-Messsystems veränderte die Oberflächeneigenschaften und somit die Interaktion mit dem umgebenden Gewebe. Die in vitro Untersuchungen zur Biokompatibilitätsprüfung erbrachten keine toxischen Eigenschaften der verwendeten Blockcopolymere. Die Untersuchung der Fibronektinadhäsion zeigte eine verminderte Anlagerung des Proteins, wodurch Immunreaktionen aufgrund des Materials ausgeschlossen werden können. Die Langzeittests der hermetischen Verkapselung des Sensorarray-Messsystems ergaben einen positiven Verlauf, so dass das verwendete System für entsprechende medizinisch relevante Anwendungen empfohlen werden kann.

Schlagwörter: biokompatible Verkapselung, implantierbarer Sensor, Hydrogele

\section{Einleitung}

Physiologische Parameter im Rahmen der Therapie von Stoffwechselkrankheiten, wie z.B. Diabetes mellitus, können durch implantierbare, drahtlose Sensorarray-Mikrosysteme kontinuierlich erfasst werden. Vorteilhafterweise werden dazu neben dem Blutzuckerspiegel auch der pH-Wert und die $\mathrm{CO}_{2}$-Konzentration gleichzeitig und kontinuierlich erfasst. Im hier untersuchten Ansatz bestehen die Sensorarrays deshalb aus mehreren Hydrogel-basierten piezoresistiven Sensoren. Die verwendeten stimuli-responsiven Hydrogele gestatten dabei die individuelle Anpassung an die jeweils zu untersuchenden Analyten. [1, 2]

\section{Methoden und Materialien}

\begin{abstract}
Sensorarray
Der Aufbau der verwendeten piezoresistiven Drucksensor-Chiparrays [3] ist in Abb. 1 schematisch dargestellt. Das Sensorprinzip basiert auf dem Quellverhalten der integrierten Hydrogele, wobei die Quellung und damit der Quelldruck der einzelnen Hydrogele unterschiedlich groß für die einzelnen Messgrößen sind. Das Messsignal der piezoresistiven Drucksensoren ist jeweils proportional zur Gelquellung. Die Signalumwandlung in eine elektrische Ausgangsspannung erfolgt über eine piezoresistive Widerstandsbrücke.
\end{abstract}




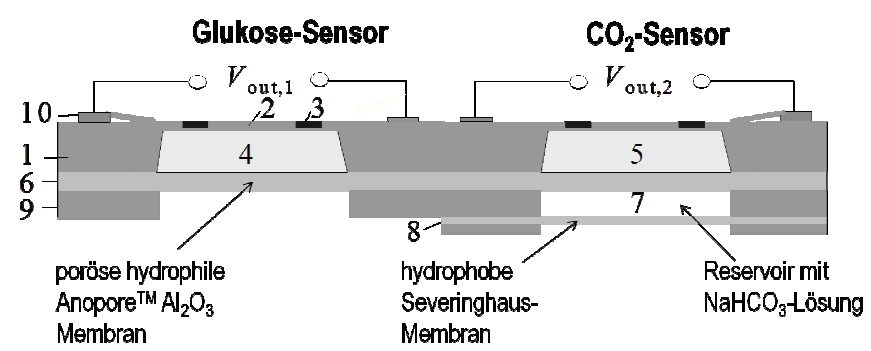

1 Si-Chip; 2 Biegeplatte; 3 mechano-elektrischer Wandler (piezoresistive Widerstandsbrücke);

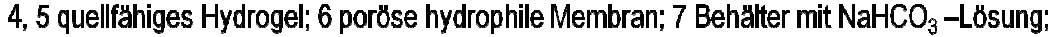
8 hydrophobe Membran; 9 Si-Substrat; 10 Kontakt

Abbildung 1: Schematischer Aufbau eines Hydrogel-basierten Sensorarrays

\section{Hydrogele}

Die in den Sensoren verwendeten Hydrogele sind Polymere mit unterschiedlichen Sensitivitäten bezüglich pH-Wert, $\mathrm{CO}_{2}, \quad$ Temperatur und Glukosekonzentration. Das Quellverhalten der Hydrogele bei Konzentrationsänderungen der Analyten im menschlichen Körper spielt eine große Rolle für die Anwendung in implantierbaren Sensoren. Der Nachweis von Glukose erfolgt im physiologischen Bereich von 2 bis $20 \mathrm{mmol} / \mathrm{l}$ Glukose. Hier werden Hydrogele mit Phenylborsäuregruppen verwendet und chemisch so modifiziert, dass die Ansprechzeiten entsprechend der Anforderungen minimal sind.

\section{Biokompatible Verkapselung}

Zur flexiblen Verkapselung des verwendeten Silizium-Chips wird Parylene C (Microelectronic Packaging Dresden $\mathrm{GmbH}$, Parylene Coating System 2000 - 150 LV) bei Raumtemperatur als ca. $5 \mu \mathrm{m}$ dünne Schicht konform abgeschieden. [4] Die Abscheidung erfolgt im Vakuum aus der Gasphase. Aufgrund dieser gasförmigen Abscheidung erreicht und beschichtet Parylene C auch Bereiche und Strukturen, welche mit flüssigkeitsbasierten Verfahren nur schwer erreichbar sind, wie z.B. scharfe Ränder und Spitzen.

Das inerte, hydrophobe, optisch transparente, polymere Beschichtungsmaterial wird durch eine zusätzliche Beschichtung mit amphiphilen Blockcopolymeren funktional ausgestattet. Die aus dem hydrophilen Polyethylenglycol (PEG) und hydrophoben Polyaminosäuren PAA bestehenden Polymere mit der Grundstruktur PEG-b-PAA $A_{1}-b / c o-$ $\mathrm{PAA}_{2}$ werden mittels metallfreier Ringöffnungspolymerisation [5] synthetisiert.
Um die Polymere an das inerte und reaktionsträge Parylene $\mathrm{C}$ zu binden, wurde ein spezielles Beschichtungskonzept entwickelt. Zunächst wird die Oberfläche mit Hilfe eines Sauerstoffplasmas aktiviert und danach mit einem silanhaltigen Haftvermittler ausgestattet. Das amphiphile Blockcopolymer wird anschließend an die Oberfläche gebunden. Eine einheitliche Schichtausbildung ist hierbei sehr wichtig, um die originären Eigenschaften der Polymere zu erhalten.

Mit Kontaktwinkelmessungen (OCA 20, DataPhysics Instruments $\mathrm{GmbH}$ ) sowie Röntgenphotoelektronenspektroskopie (XPS, ESCA 5700, Physical Electronics PHI) erfolgt der Nachweis der Polymeranbindung an die Oberfläche.

\section{In vitro-Experimente}

Zum Nachweis der Bioverträglichkeit der entwickelten Sensorverkapselung aus Parylene C und den amphiphilen Blockcopolymeren wurden humane Fibroblasten (Coriell Institute, NJ, USA) verwendet. Für die in vitro-Versuche wurde mit Parylene $\mathrm{C}$ und Blockcopolymeren beschichteten Glasdeckgläschen (Dicke $18 \mathrm{~mm}$ ) gearbeitet. Die Inkubation erfolgte in entsprechenden Zellkulturgefäßen mit einer Zelldichte von 40000 Zellen $/ \mathrm{ml}$. Die Zellen wurden für $72 \mathrm{~h}$ bei $37{ }^{\circ} \mathrm{C}, 80 \%$ Luftfeuchte und $5 \% \mathrm{CO}_{2}$ in einem Brutschrank kultiviert. Die Ermittlung der Zellviabilität erfolgte mittels Cell Titer Blue Assay (Promega GmbH).

Zudem wurde die Apoptoserate mit Hilfe von Propidiumiodid (PI) ermittelt. Die gefärbten Zellen wurden wie beschrieben kultiviert und danach mit PI für 30 min im Dunklen bei $37^{\circ} \mathrm{C}$ inkubiert, mit 
Phosphat gepufferter Salzlösung gewaschen und am FACScan (Becton Dickinson) mittels Argonlaser (488 nm) detektiert.

\section{Proteinadhäsion}

Die Interaktion von implantierbaren Materialien und dem Gewebe läuft neben der zellulären Ebene vor allem auch auf der Proteinebene ab. Die Anlagerung von adhäsionsvermittelnden Proteinen, wie Fibronektin, fördern die Zellanheftung an das Material, aber auch unerwünschte Immunreaktionen. Die Adhäsion wird mit Hilfe von fluoreszenzmarkierten Proteinen quantifizierbar. Hierfür wurden die beschichteten Proben, die auch für die In vitro Versuche verwendet werden, für $30 \mathrm{~min}$ bei $37^{\circ} \mathrm{C}$ mit dem fluoreszenzmarkierten Fibronektin HiLyte 488 (BIOMOL $\mathrm{GmbH}$ ) inkubiert und drei Mal mit PBS gewaschen. Die Fluoreszenzintensität wurde am Mikroplattenleser (Infinite 200 PRO, Tekkan) erfasst.

Zusätzlich wurde die Interleukin-8- (IL-8) Expression der humanen Fibroblasten durch einen ELISA- (Enzyme Linked Immunosorbent Assay) Test untersucht (BIOMOL GmbH). Die Zellen wurden wie beschrieben kultiviert. Nach Erreichen von $80 \%$ Konfluenz wurden die Überstände gesammelt und bis zum Versuch bei $20{ }^{\circ} \mathrm{C}$ gelagert. Als Kontrolle wurde ein Teil der Proben mit Lipopolysacchariden (LPS) stimuliert. Diese lösen Entzündungsreaktionen aus und steigern dadurch die IL-8-Expression der verwendeten humanen Fibroblasten. Die Auswertung erfolgte mittels 4-ParameterRegression.

\section{Ergebnisse}

Die mehrschichtige Verkapselung der implantierbaren Biosensoren verändert die Oberflächeneigenschaften. Kontaktwinkelmessungen zeigen die Veränderung über den Verlauf des Beschichtungsprozesses von Parylene C mit dem amphiphilem Blockcopolymer (Abb. 2).
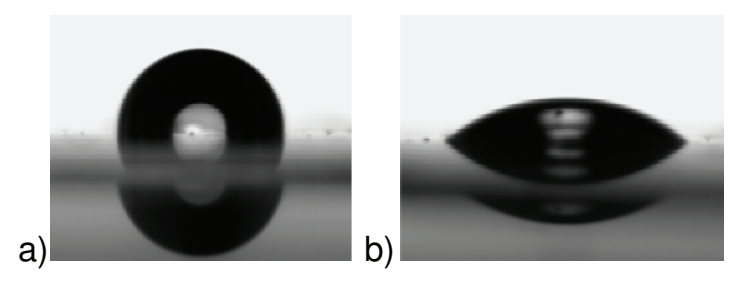

Abbildung 2: Kontaktwinkelmessungen an Wassertropfen auf a) Parylene C, $\theta=90,0^{\circ}$, b) Parylene C/amphiphiles Blockcopolymer, $\theta=$ $44,3^{\circ}$

Die Analysen mit XPS zeigen, dass sich die Anteile an Kohlenstoff, Stickstoff sowie Sauerstoff, Silizium und Chlor durch die Beschichtung verändern. Die Funktionalisierung der Oberfläche mit dem Blockcopolymer erhöht den Sauerstoffanteil und verschiebt das Verhältnis zum Kohlenstoffanteil. Dies wird durch den hohen Kohlen- und Sauerstoffanteil im Polymer verursacht (Abb. 3).

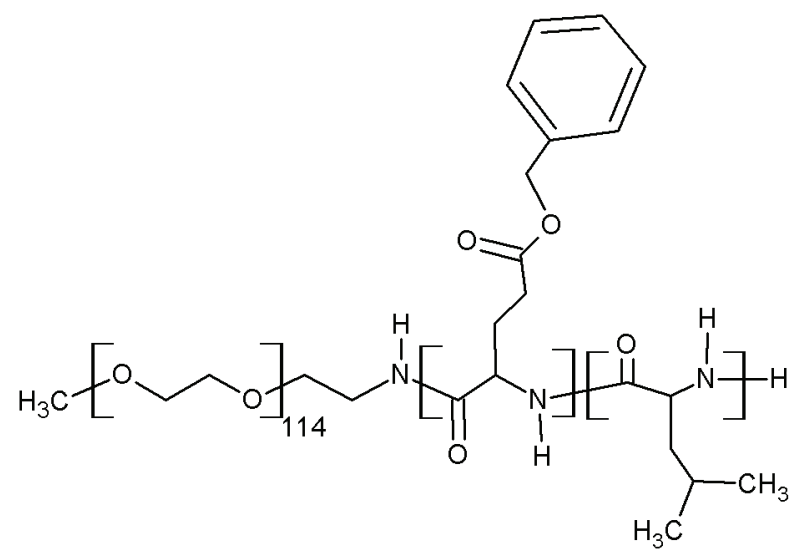

Abbildung 3: Strukturformel des verwendeten Blockcopolymers; $P G_{114}-b-p(L-G l u)_{40}-b-p(L-L e u)_{10}$

Die in vitro-Versuche mit dem Cell Titer Blue Assay (Abb. 4) zeigen eine ca. $20 \%$ geringere Zellviabilität der Polymerprobe und eine ca. $40 \%$ geringere Zellviabilität bei Parylene $\mathrm{C}$ im Vergleich zur Kontrollprobe (Zellkultur-Well). Die geringere Viabilität bei der Polymerprobe ist laut DIN EN ISO 10993-5:2009 für Medizinprodukte als nicht toxisch für die humanen Fibroblasten und folglich als zellverträglich einzustufen. Weiterhin kann man eine deutliche Verbesserung im Vergleich zu Parylene $\mathrm{C}$ erkennen. 


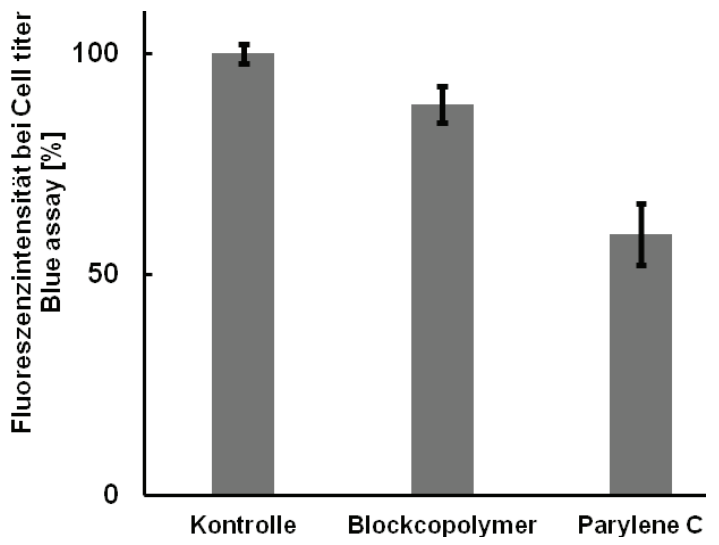

Abbildung 4: Fluoreszenzintensität als $\mathrm{Ma} B$ für die Zellviabilität bei direktem Kontakt mit dem zu untersuchenden Material (Parylene C, Blockcopolymer)

In Abbildung 5 sind die Anteile an vitalen, apoptotischen und nekrotischen Zellen nach der Kultivierung auf Parylene $\mathrm{C}$ und den mit Blockcopolymer beschichteten Proben dargestellt. Die höhere Apoptoserate bei den nur mit Parylene $C$ beschichteten Proben bestätigt die Ergebnisse der Untersuchungen zur Zellviabilität. Die Polymerproben zeigen nur eine geringe Erhöhung der Apoptoserate und zeigen auch hier die Bioverträglichkeit des Materials.

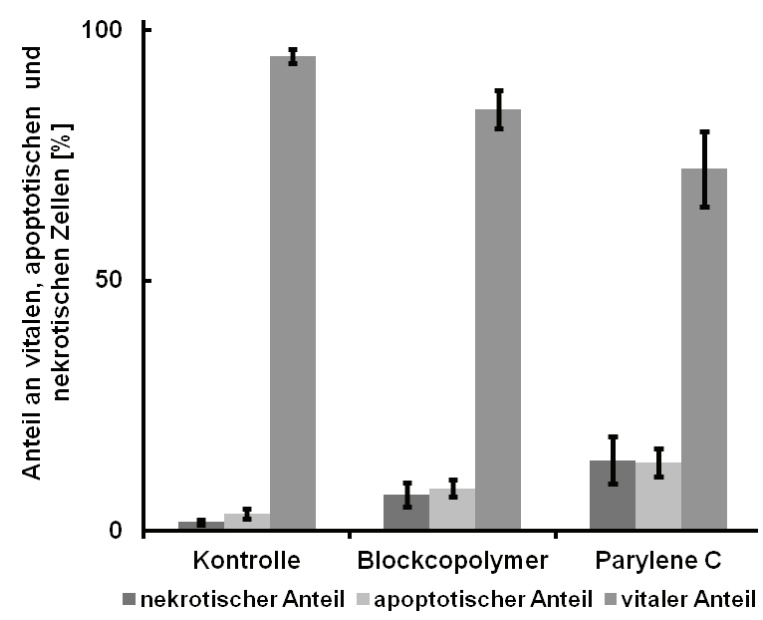

Abbildung 5: Vitale, apoptotische und nekrotische Zellen nach Färbung mit PI und Detektion am FACScan

Die Untersuchungen zur Fibronektinadhäsion sind in Abbildung 6 zusammengefasst. Die Blockcopolymere als biokompatible Beschichtung vermindern die Anlagerung von Fibronektin um mehr als $50 \%$ im Vergleich zur Kontrollprobe.

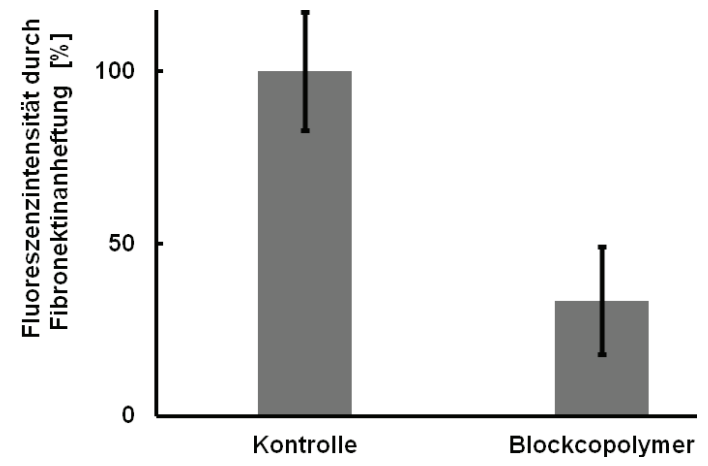

Abbildung 6: Fluoreszenzintensität als Maß der Fibronektinadhäsion der mit Polymer $\mathrm{A}$ beschichteten Proben nach Inkubation mit fluoreszenzmarkiertem Fibronektin HiLyte 488

Der IL-8-ELISA zeigt, dass die humanen Fibroblasten durch die Kultivierung auf den unterschiedlich beschichteten Probenoberflächen (Parylene C, Blockcopolymer) keine erhöhte IL-8Ausschüttung aufweisen (Abb. 7). Nur die LPSstimulierte Kontrolle weist hohe IL-8-Werte auf, wie sie bei einer Entzündungsreaktion vorkommen können.

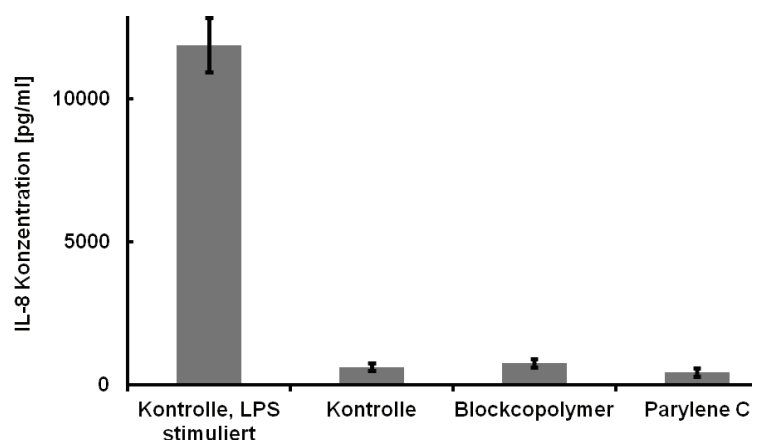

Abbildung 7: IL-8-Expression von humanen Fibroblasten nach inkubation auf Parylene $\mathrm{C}$ und dem Blockcopolmer sowie den entsprechenden Kontrollproben

\section{Diskussion}

Die Beschichtung des SensorarrayMesssystems mit Parylene $\mathrm{C}$ und unterschiedlichen amphiphilen Blockcopolymeren verändert die Oberflächeneigenschaften. Es konnten Veränderungen im Kontaktwinkel nachgewiesen werden. Dieser spielt auch bei 
Implantaten eine große Rolle, um die Adhäsion von Proteinen und Zellen zu beeinflussen.

Das inerte Parylene $C$ ist sehr reaktionsträge und nur schwer funktionell auszustatten. Die Anbindung der Blockcopolymere an Parylene C wurde mit Hilfe des entwickelten Beschichtungskonzeptes realisiert und optimiert.

Die polymeren Beschichtungen geben den Implantaten proteinähnliche Eigenschaften, die diese im Körper als nicht sichtbar erscheinen lassen und Entzündungsreaktionen mindern. Dieser Effekt ist auch hier nachgewiesen worden. Die Untersuchungen zur Biokompatibilität weisen zudem keine toxischen Eigenschaften von den verwendeten Blockcopolymeren auf.

Demnach werden durch die mehrschichtige Verkapselung einerseits der Schutz der empfindlichen elektronischen Komponenten des Messsystems und andererseits eine hohe Biokompatibilität der Oberfläche erreicht.

Die Verkapselung kann für viele funktionale Implantate Verwendung finden. Die spezielle Synthese der Blockcopolymere erlaubt zudem auch eine Variation in den entsprechenden Polyaminosäuren und somit eine Anpassung an verschiedenste Anwendungsgebiete.

\section{Literatur:}

[1] GERLACH, G. ; ARNDT, K-F. (Hrsg.): Hydrogel Sensors and Actuators, Springer Series on Chemical Sensors and Biosensors. Vol. 6. Springer-Verlag. 2009.

[2] LIN, G. ; CHANG, S. ; HAO, H. ; TAITHREDDY, P. ; ORTHNER, M. MAGDA, J. ; SOLZBACHER, F.: Osmotic swelling response of smart hydrogels suitable for chronically implantable glucose sensors. In: Sensors and Actuators B: Chemical 144 (2010), S. 332-336

[3] GUENTHER, M. ; GERLACH, G. ; WALLMERSPERGER, T. ; AVULA, M.N. ; CHO, S.H. ; XIE, X. ; DEVENER, B.V. ; SOLZBACHER, F. ; TATHIREDDY, P. ; MAGDA, J.J. ; SCHOLZ, C. ; OBEID, R. ; ARMSTRONG, T.: Smart Hydrogel-Based Biochemical Microsensor Array for Medical Diagnostics. In: Advances in Science \& Technology 85 (2013), S. 47-52.

[4] KIRSTEN S. ; WETTERLING, J. ; UHLEMANN, J. ; WOLTER, K. ; ZIGLER, S.: Barrier properties of polymer encapsulation materials for implantable microsystems. In: 2013 IEEE
XXXIII International Scientific Conference Electronics and Nanotechnology (ELNANO). Kiev, Ukraine, S. 269-272.

[5] OBEID, R. ; SCHOLZ, C.: Synthesis and selfassembly of well-defined poly(amino acid) endcapped poly(ethylene glycol) and poly(2-methyl2-oxazoline). In: Biomacromolecules 12 (2011), Nr. 10, S. 3797-3804.

\section{Danksagung}

Die Autoren danken Sergej Zigler von der Fa. Microelectronic Packaging Dresden für die Parylene C-Beschichtung sowie der Deutschen Forschungsgemeinschaft (DFG) für die finanzielle Unterstützung. 